\title{
Surrogate endpoints for overall survival in advanced non-small-cell lung cancer patients with mutations of the epidermal growth factor receptor gene
}

\author{
REIKO YOSHINO $^{1 *}$, HISAO IMAI $^{2 *}$, KEITA MORI $^{3}$, KOUSUKE TAKEI $^{1}$, MAI TOMIZAWA $^{1}$, KYOICHI KAIRA $^{4}$, \\ AKIHIRO YOSHII ${ }^{1}$, YOSHIO TOMIZAWA ${ }^{1}$, RYUSEI SAITO $^{1}$ and MASANOBU YAMADA ${ }^{2}$ \\ ${ }^{1}$ Department of Respiratory Medicine, National Hospital Organization Nishigunma Hospital, Shibukawa, Gunma 377-8511; \\ ${ }^{2}$ Department of Medicine and Molecular Science, Gunma University Graduate School of Medicine, Maebashi, \\ Gunma 371-8511; ${ }^{3}$ Clinical Trial Coordination Office, Shizuoka Cancer Center, Nagaizumi, Shizuoka 411-8777; \\ ${ }^{4}$ Department of Oncology Clinical Development, Gunma University Graduate School of Medicine, \\ Maebashi, Gunma 371-8511, Japan
}

Received May 6, 2014; Accepted June 11, 2014

DOI: $10.3892 / \operatorname{mco} .2014 .334$

\begin{abstract}
Subsequent therapies confound the ability to discern the effect of first-line chemotherapy on overall survival (OS). We investigated whether progression-free survival (PFS), post-progression survival (PPS) and tumor response were valid surrogate endpoints for OS following first-line chemotherapy in individual patients with advanced non-small-cell lung cancer (NSCLC) harboring sensitive epidermal growth factor receptor gene mutations. We retrospectively analyzed 35 patients with advanced NSCLC treated with first-line gefitinib. The associations of PFS, PPS and tumor response with OS were analyzed. PPS was found to be strongly correlated with OS, unlike PFS and tumor shrinkage. The factors significantly associated with PPS were performance status (PS) after first-line treatment, best response to second-line treatment and number of regimens used after disease progression. PPS may be a surrogate for OS in this patient population and further therapy after disease progression following first-line chemotherapy may significantly affect OS. However, a larger study is required to validate these results.
\end{abstract}

\section{Introduction}

Lung cancer is the most common cause of cancer-related mortality worldwide, with non-small-cell lung cancer

Correspondence to: Dr Hisao Imai, Department of Medicine and Molecular Science, Gunma University Graduate School of Medicine, 3-39-15 Showa-machi, Maebashi, Gunma 371-8511, Japan

E-mail:m06701014@gunma-u.ac.jp

*Contributed equally

Key words: non-small-cell lung cancer, epidermal growth factor receptor mutation, overall survival, post-progression survival, tumor response, progression-free survival
(NSCLC) accounting for $\sim 85 \%$ of all lung cancer cases (1). Overall survival (OS) is considered the most reliable endpoint in cancer studies and is generally the preferred endpoint for survival studies (2). OS is precise, easily measured and documented by the date of death. Surrogate endpoints, such as tumor response and progression-free survival (PFS), are also valuable in oncology clinical trials, since they can be measured earlier, are easier to assess compared to 'true' endpoints and the events are more frequent.

With the growing number of drugs and combination therapies available for the treatment of NSCLC, the effect of first-line chemotherapy on OS may be confounded by subsequent therapies (3). Indeed, in a recent randomized trial on NSCLC patients, an improvement in PFS did not necessarily result in improved OS (4). A number of compounds are currently available for second- and third-line chemotherapy for diseases such as breast, ovarian and colorectal cancer (5-7), as well as advanced NSCLC. Although PFS following first-line chemotherapy is not a validated surrogate endpoint for OS, post-progression survival (PPS) has been shown to be strongly associated with OS after first-line chemotherapy for advanced NSCLC $(8,9)$. PPS has also become strongly associated with OS over the last decade (2002-2012), in which molecularly targeted agents, such as gefitinib and erlotinib, have been used to treat advanced NSCLC $(8,9)$. PPS is calculated as follows: OS=PFS+PPS (2).

The effect on survival of therapies administered after disease progression is of interest at the individual patient level. Past analysis of individual-level data suggested that PPS was a surrogate for OS in patients with advanced non-squamous NSCLC with unknown oncogenic driver mutations and, therefore, limited options for subsequent chemotherapy (10). However, it is unknown whether this applies to advanced NSCLC patients with epidermal growth factor receptor gene $(E G F R)$ mutations sensitive to targeted therapy. Therefore, examination of individual-level data to determine whether PFS, PPS and tumor response are valid surrogate endpoints for OS after first-line therapy in these patients may be of clinical value. 
Previous clinical trials have identified gefitinib, an EGFR tyrosine kinase inhibitor (EGFR-TKI), as a first-line treatment option for patients with NSCLC with sensitive EGFR mutations (11-13). Although several patients achieve initial clinical remission or disease control with first-line chemotherapy, the majority experience subsequent disease progression and death. We examined first-line gefitinib chemotherapy, as it is the standard first-line chemotherapy for advanced NSCLC with EGFR mutations. Patients with NSCLC harboring an EGFR mutation who were treated with gefitinib, platinum and pemetrexed or docetaxel exhibited a median survival of $\sim 3$ years (14). For advanced NSCLC patients with sensitive EGFR mutations, the OS time is longer and there are additional options for subsequent chemotherapy.

In this study, we aimed to determine the associations of PFS, PPS and tumor response with OS in patients with advanced NSCLC harboring sensitive EGFR mutations. We also assessed the prognostic value of baseline and tumor characteristics for PPS.

\section{Patients and methods}

Patients. This study included 46 patients with advanced NSCLC harboring sensitive EGFR mutations who were treated with first-line gefitinib between January, 2006 and June, 2012. The eligibility criteria were histologically or cytologically proven NSCLC, unresectable stage IIIB/IV disease, a tumor with a drug-sensitive EGFR mutation (exon 18 G719X, exon 19 deletion, or exon 21 L858R) and continuous gefitinib treatment. Tumor response was not evaluated in 1 patient and PFS data were censored in 10 patients. To ensure patient background uniformity, these 11 patients were excluded from the analysis. Therefore, 35 patients were retrospectively analyzed (Fig. 1). Genomic DNA was extracted from tumor samples and EGFR mutations in exons 18-21 were analyzed as previously described $(15,16)$.

The study protocol was approved by the Institutional Review Board of the National Hospital Organization Nishigunma Hospital.

Response to treatment. The patients received first-line gefitinib (250 mg per os, once daily), which continued until disease progression, development of intolerable toxicity, or withdrawal of consent. All the patients were EGFR-TKI naïve.

The best overall response and maximum tumor shrinkage were recorded as tumor responses. Radiographic tumor responses were evaluated according to the Response Evaluation Criteria in Solid Tumors, version 1.1 (17) as follows: complete response (CR), disappearance of all target lesions; partial response (PR), decrease in the sum of the target lesion diameters by $\geq 30 \%$ compared to baseline diameters; progressive disease (PD), increase of $\geq 20 \%$ in the sum of the target lesion diameters compared to the smallest sum during the study; and stable disease (SD), insufficient shrinkage or expansion to qualify as PR or PD. PFS was calculated from the start of treatment until PD or death from any cause. OS was recorded from the first day of treatment until death or was censored on the date of the last follow-up. PPS was recorded as the time from tumor progression until death or was censored on the date of the last follow-up.
Table I. Baseline patient characteristics.

\begin{tabular}{|c|c|}
\hline Characteristics & $\begin{array}{l}\text { Patient no. } \\
\qquad(n=35)\end{array}$ \\
\hline \multicolumn{2}{|l|}{ Gender } \\
\hline Male/female & $11 / 24$ \\
\hline \multicolumn{2}{|l|}{ Age at treatment, years } \\
\hline Median (range) & $67(45-88)$ \\
\hline \multicolumn{2}{|l|}{ Performance status } \\
\hline $0 / 1 / \geq 2$ & $15 / 17 / 3$ \\
\hline \multicolumn{2}{|l|}{ Histology } \\
\hline Adenocarcinoma/others & $35 / 0$ \\
\hline \multicolumn{2}{|l|}{ Stage } \\
\hline IIIB/IV & $3 / 32$ \\
\hline \multicolumn{2}{|l|}{ Mutation status } \\
\hline Exon 19 del/exon 21 L858R/others & $20 / 15 / 0$ \\
\hline \multicolumn{2}{|l|}{ Administration period, years } \\
\hline$<1 / \geq 1$ & $20 / 15$ \\
\hline \multicolumn{2}{|l|}{ Number of regimens after progression } \\
\hline $0 / 1 / 2 / 3 / 4 / 5 / \geq 6$ & $11 / 6 / 6 / 3 / 5 / 1 / 3$ \\
\hline Median (range) & $2(0-10)$ \\
\hline \multicolumn{2}{|l|}{ Sum of target lesion diameters, $\mathrm{mm}$} \\
\hline Median (range) & $25(10-85)$ \\
\hline
\end{tabular}

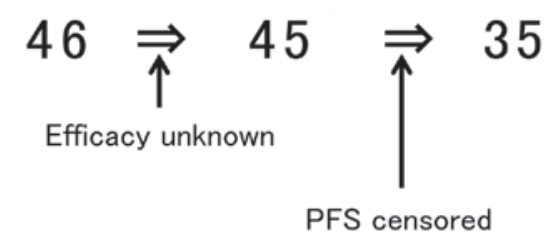

Figure 1. Flow chart showing patient selection. The patients received epidermal growth factor receptor-tyrosine kinase inhibitor first-line chemotherapy between January, 2006 and June, 2012. PFS, progression-free survival.

Statistical analyses. To determine whether PFS, PPS and tumor shrinkage were correlated with OS, we used Spearman's rank correlation analysis and linear regression analysis. To identify the prognostic factors for PPS, we applied the proportional hazards model with a stepwise regression procedure. Hazard ratios (HRs) and 95\% confidence intervals (95\% CIs) were estimated. As the HR is defined for a 1-unit difference, certain factors were converted to an appropriate scale. PPS values were compared using the log-rank test. $\mathrm{P} \leq 0.05$ was considered to indicate statistically significant differences. The two-tailed significance level was set at 0.05 . All the statistical analyses were performed using JMP software for Windows, version 9.0 (SAS Institute, Cary, NC, USA).

\section{Results}

Patient characteristics and treatment efficacy. Of the 35 patients included in the analysis, 25 succumbed to their disease; the median follow-up was 21.0 months (range, 
Table II. Chemotherapeutic regimens administered after disease progression following first-line chemotherapy.

\begin{tabular}{lccc}
\hline Regimens & Second-line & $\geq$ Third-line & Total \\
\hline Platinum combination & 19 & 7 & 26 \\
Docetaxel & 0 & 9 & 9 \\
Pemetrexed & 2 & 2 & 4 \\
Erlotinib & 3 & 5 & 8 \\
Gefitinib rechallenge & 0 & 6 & 6 \\
S1 & 0 & 7 & 7 \\
Gemcitabine & 0 & 4 & 4 \\
Amrubicin & 0 & 2 & 2 \\
Others & 0 & 5 & 5 \\
Investigational agent & 0 & 0 & 0 \\
\hline
\end{tabular}
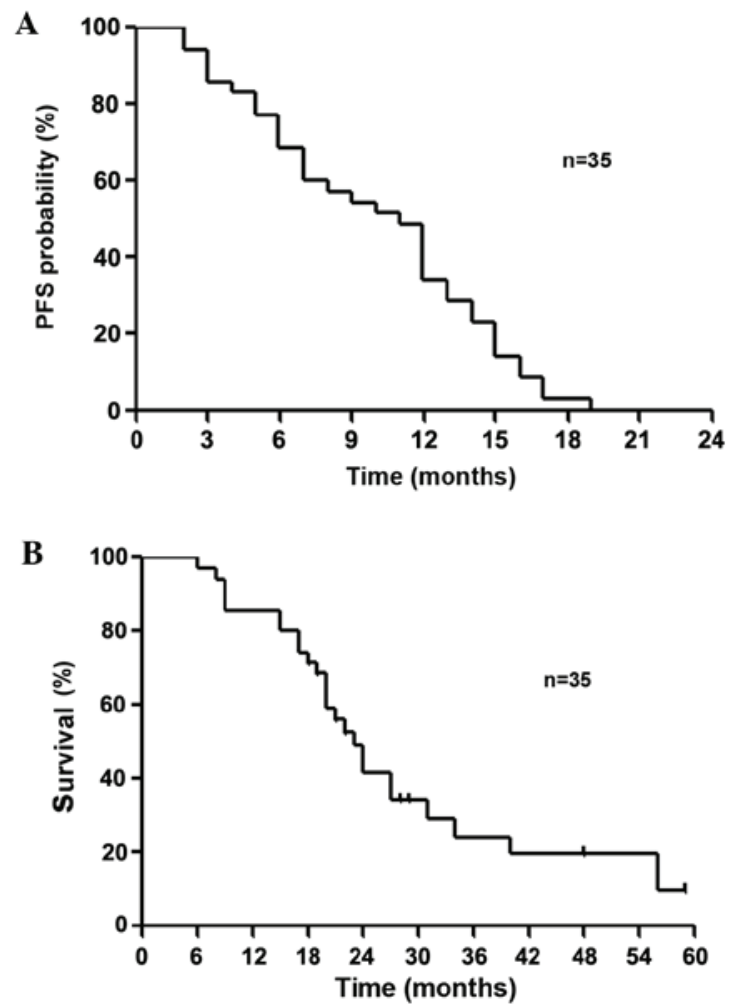

Figure 2. Kaplan-Meier plot showing (A) progression-free survival (PFS); median PFS, 11.0 months and (B) overall survival (OS); median OS, 23.0 months. The median follow-up time was 21.0 months.

6.0-56.0 months). The patients' median age was 67 years (range, $45-88$ years). The patient characteristics are summarized in Table I. Target lesions were not evaluated in 1 patient.

Of the 35 patients, 2, 20, 12 and 1 exhibited CR, PR, SD and PD, respectively. The response rate was $62.8 \%$ and the disease control rate was $97.1 \%$. After disease progression following first-line chemotherapy, 11 of the 35 patients did not receive additional chemotherapy, whereas the remaining 24 did. Among the 35 patients, the median number of follow-up therapeutic regimens was 2 (range, $0-10$ regimens). The chemotherapeutic regimens employed after disease progression are
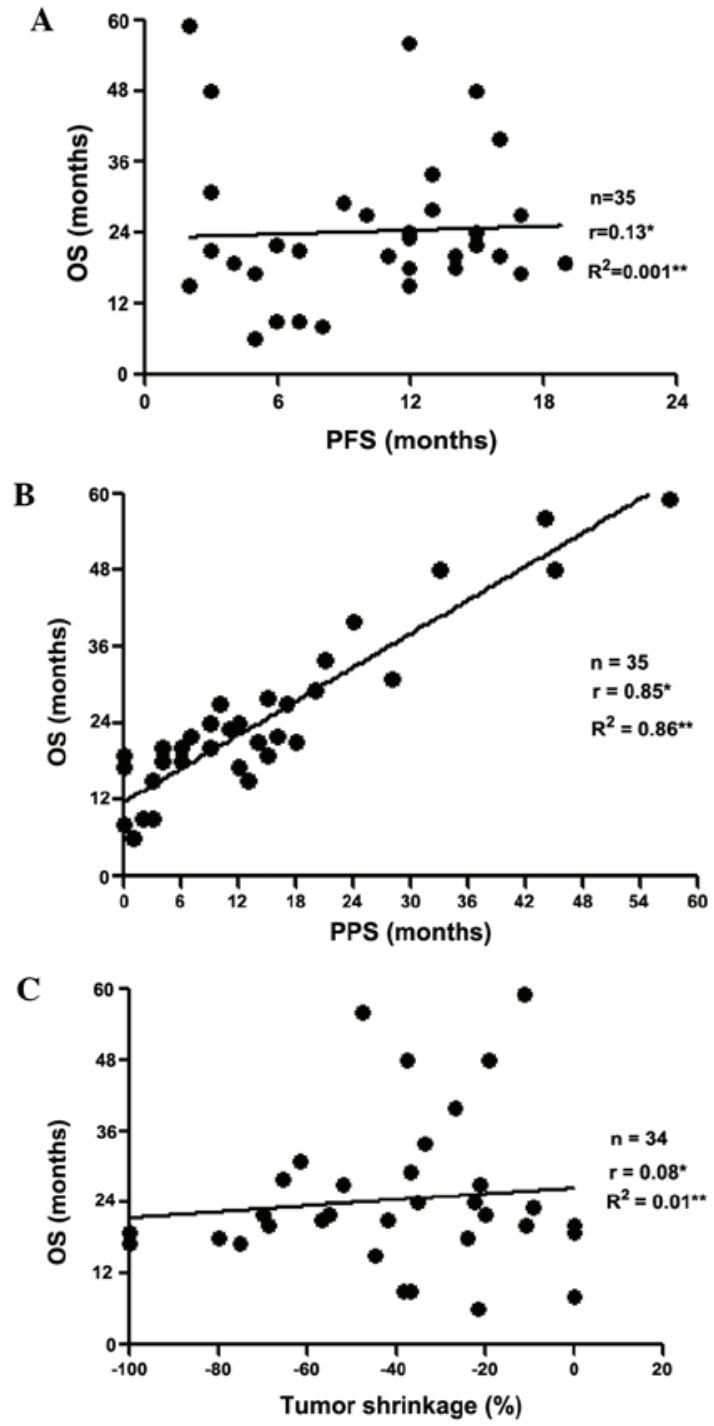

Figure 3. (A) Correlation between overall survival (OS) and progression-free survival (PFS). (B) Correlation between OS and post-progression survival (PPS). (C) Correlation between OS and tumor shrinkage. "Spearman's rank correlation coefficient; ${ }^{* *}$ linear regression.

listed in Table II. Platinum combination chemotherapy was the most common second-line treatment and docetaxel was the most common treatment following second-line chemotherapy. The median PFS and OS were 11.0 and 23.0 months, respectively (Fig. 2).

Association between OS and PFS, PPS and tumor shrinkage. The associations between OS and PFS, PPS and tumor shrinkage are shown in Fig. 3A, B and C, respectively. Based on Spearman's rank correlation coefficients and linear regression, PPS was found to be strongly associated with OS $(\mathrm{r}=0.85$, $\left.\mathrm{P}<0.05, \mathrm{R}^{2}=0.86\right)$, unlike PFS $\left(\mathrm{r}=0.13, \mathrm{P}=0.45, \mathrm{R}^{2}=0.001\right)$ and tumor shrinkage $\left(\mathrm{r}=0.08, \mathrm{P}=0.61, \mathrm{R}^{2}=0.01\right)$.

Factors affecting PPS. The univariate analysis demonstrated that the factors associated with PPS $(\mathrm{P}<0.05)$ were age at the beginning of first-line treatment; performance status (PS) at the beginning and at the end of first-line treatment and at the beginning of second-line treatment; the best response to 
Table III. Univariate Cox regression analysis of the association between baseline patient characteristics and post-progression survival.

\begin{tabular}{lcrr}
\hline & \multicolumn{2}{c}{ Post-progression survival } \\
\cline { 2 - 4 } Characteristics & Hazard ratio & $95 \%$ CI & P-value \\
\hline Gender (male vs. female) & 1.53 & $0.57-3.73$ & 0.37 \\
Age at the beginning of first-line treatment & 1.07 & $1.02-1.12$ & $<0.01$ \\
PS at the beginning of first-line treatment & 1.73 & $1.05-2.69$ & 0.03 \\
Stage (IIIB vs. IV) & 2.03 & $0.47-6.06$ & 0.29 \\
EGFR mutation status (exon 19 del vs. exon 21 L858R) & 0.48 & $0.21-1.08$ & 0.07 \\
Sum of longest diameter of target lesions & 1.01 & $0.98-1.03$ & 0.24 \\
Best response at first-line treatment & & & 0.36 \\
PR vs. non-PR & 0.69 & $0.30-1.54$ & 0.24 \\
Non-PD vs. PD & 0.22 & $0.04-4.22$ & $<0.01$ \\
PS at the end of first-line treatment & 2.01 & $1.38-2.96$ & 0.07 \\
Age at the beginning of second-line treatment & 1.06 & $0.99-1.15$ & 0.02 \\
PS at the beginning of second-line treatment & 3.6 & $1.20-13.6$ & 0.07 \\
Best response to second-line treatment & & & $<0.01$ \\
PR vs. non-PR & 0.33 & $0.07-1.09$ & $<0.01$ \\
$\quad$ Non-PD vs. PD & 0.13 & $0.03-0.46$ & $0.41-0.76$ \\
Number of regimens after progression beyond first-line chemotherapy & 0.57 & &
\end{tabular}

95\% CI, 95\% confidence interval; PS, performance status; EGFR, epidermal growth factor receptor; PR, partial response; PD, progressive disease.

Table IV. Multivariate Cox regression analysis of the association between post-progression survival and age at the beginning of first-line treatment, PS at the end of first-line treatment, best response to second-line treatment and number of regimens employed after progression beyond first-line chemotherapy.

\begin{tabular}{lcrr}
\hline & \multicolumn{3}{c}{ Post-progression survival } \\
\cline { 2 - 4 } Variables & Hazard ratio & $95 \% \mathrm{CI}$ & P-value \\
\hline Age at the beginning of first-line treatment & 1.03 & $0.93-1.14$ & 0.53 \\
PS at the end of first-line treatment & 3.67 & $1.00-17.9$ & 0.04 \\
Best response to second-line treatment (non-PD vs. PD) & 0.11 & $0.01-0.54$ & $<0.01$ \\
Number of regimens after progression beyond first-line chemotherapy & 0.47 & $0.24-0.75$ & $<0.01$
\end{tabular}

PS, performance status; $95 \%$ CI, 95\% confidence interval; PD, progressive disease.

second-line treatment; and the number of regimens employed after disease progression following first-line chemotherapy (Table III). Subsequently, the multivariate analysis revealed that the clinical factors affecting PPS were the PS at the end of the first-line treatment, best response to second-line treatment (non-PD vs. PD) and number of regimens employed after disease progression following first-line chemotherapy $(\mathrm{P}<0.05$, Table IV). Log-rank tests were used to confirm that these 3 factors were significantly associated with PPS (log-rank test, $\mathrm{P}<0.05$; Fig. 4). Patients with PS 0 at the end of first-line treatment had a PPS of 21.0 months, patients with PS 1 had a PPS of 16.5 months, patients with PS 2 had a PPS of 2.0 months, patients with PS 3 had a PPS of 4.0 months and patients with PS 4 had an undefined PPS (log-rank test, $\mathrm{P}=0.04$; Fig. 4A). Furthermore, patients with non-PD had a median PPS of 24.0 months, whereas their PD counterparts had a median PPS of 7.0 months (log-rank test, P<0.001; Fig. 4B). The PPS for patients who did not receive additional treatment regimens after disease progression following first-line chemotherapy was 6.5 months; with 1 additional regimen, the PPS was 4.5 months; and with $\geq 2$ regimens, the PPS was 21.0 months (log-rank test, $\mathrm{P}<0.001$; Fig. $4 \mathrm{C}$ ). These results remained 
A

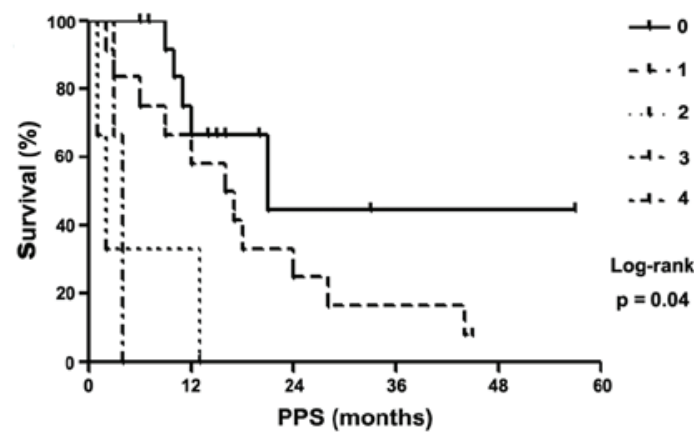

B

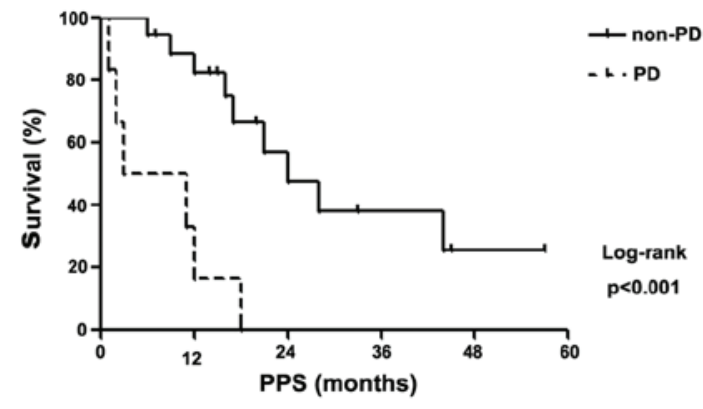

C

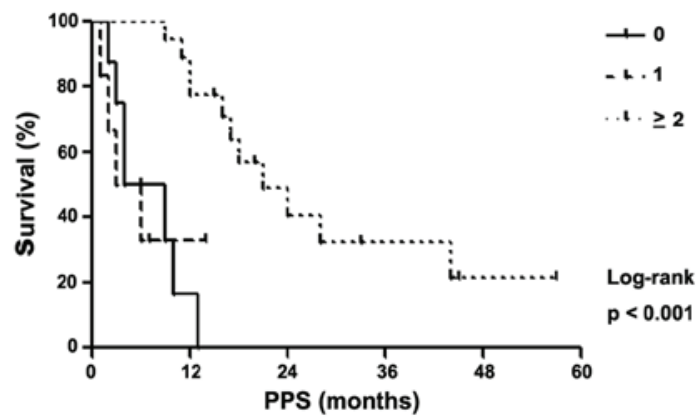

Figure 4. Kaplan-Meier plots. (A) Post-progression survival (PPS) according to performance status (PS) at the beginning of second-line treatment. PS 0: median, 21.0 months; PS 1: median, 16.5 months; PS 2: median, 2.0 months; PS 3: median, 4.0 months; PS 4: undefined. (B) PPS according to the best response following second-line treatment. Non-progressive disease (PD): median, 24.0 months; PD: median, 7.0 months (C) PPS according to the number of regimens administered after progression. No regimens: median, 6.5 months; 1 regimen: median, 4.5 months; $\geq 2$ regimens: median, 21.0 months.

consistent following adjustment in the Cox proportional hazards model (Table IV).

\section{Discussion}

We investigated the associations between OS and PFS, PPS and tumor shrinkage at the individual patient level. PPS was found to be strongly associated with OS, unlike PFS and tumor shrinkage. Additionally, PPS was affected by the PS at the end of first-line treatment, best response to second-line treatment (non-PD vs. PD) and number of regimens employed after disease progression following first-line chemotherapy.

The validity of surrogate endpoints has been determined by previous meta-analyses $(18,19)$. Biostatisticians have also proposed measures for validating surrogate endpoints $(20,21)$. Tumor response and PFS are potential surrogate endpoints for OS in extensive-stage small-cell lung cancer (22), although their validity in advanced NSCLC is controversial (23). Broglio and Berry (2) recently focused on PPS, which they defined as survival post-progression (OS minus PFS), in a hypothetical clinical trial situation; their study hypothesized that treatment affected PFS, but not PPS. Recently, PPS was found to be strongly associated with OS after first-line chemotherapy for advanced NSCLC $(8,9)$.

Our results do not correspond to those of certain previous studies indicating that tumor response and PFS may be surrogate endpoints for OS in advanced NSCLC $(24,25)$. In our patients with advanced NSCLC harboring EGFR mutations who received first-line gefitinib, PFS and tumor response did not reflect OS. PPS was more closely associated with OS rather than PFS; the association between PPS and OS was linear (Fig. 3A and B). PPS accounted for a large part of OS, suggesting that chemotherapy was too weak for PFS to prolong OS. Therefore, in clinical trials with patients expected to have a short PFS after first-line chemotherapy, such as those in our study, we must control for factors that affect the PPS.

A previous clinical trial for advanced NSCLC demonstrated that a long PPS was associated with a good PS, the use of first-line monotherapy and a molecularly targeted agent (8). However, no studies have yet investigated individual patient data to determine factors that affect PPS in advanced NSCLC with EGFR mutations. We aimed to determine whether baseline factors were prognostic for PPS and found that the PS at the end of first-line treatment, best response to second-line treatment and number of regimens employed after disease progression were strongly associated with PPS; this was confirmed by using log-rank tests. To the best of our knowledge, this study is the first to report individual-level factors that affect PPS in patients with advanced NSCLC with targeted therapy-sensitive EGFR mutations. Our findings suggest that patients with a good PS at the end of first-line treatment are able to achieve SD after disease progression. These patients are also likely to be able to continue chemotherapy and achieve a prolonged PPS, which is associated with a prolonged OS. The large number of treatment regimens used after disease progression is likely the result of the increasing number of active compounds, such as docetaxel, pemetrexed, S1, gemcitabine, amrubicin and erlotinib, which are currently available for second- and third-line chemotherapy for advanced NSCLC (Table II). Furthermore, re-administration of gefitinib is reported to be effective and is therefore a treatment option for patients with NSCLC who are initially responsive to gefitinib, but acquire resistance following subsequent chemotherapy (26).

This study had several limitations. First, the sample size was small. Only a small number of advanced NSCLC patients harboring therapy-sensitive EGFR mutations who were treated with first-line gefitinib were seen at our single institution, leading to this limitation. Our sample size was also limited by our attempt to analyze patients with similar backgrounds. However, our institution treats a relatively large number of such cases and our practices and policies are largely unified, since this is a single institution. Understanding the nature of the sources of bias in this study ensures that the results are meaningful. A future study that includes a larger patient cohort is required. Second, we were unable to thoroughly evaluate the treatments administered after disease progression following second-line chemotherapy. However, our results are meaningful, as only a limited number of patients received third-line or subsequent 
chemotherapy. Third, individual physicians decided on the date on which responses were recorded, possibly introducing variance into the measurements of PFS and tumor response. Fourth, the patients in this study received gefitinib, and not erlotinib, as first-line chemotherapy. However, this reflects the clinical setting, in which the majority of patients are treated with gefitinib.

In conclusion, using individual-level data, we observed that PPS, but not PFS or tumor response, was a surrogate for OS in patients with advanced NSCLC with targeted therapy-sensitive EGFR mutations. Additionally, a PFS advantage was not associated with increased OS, whereas PPS exerted a more significant influence on OS. Furthermore, the PS at the end of first-line treatment, best response to second-line treatment (non-PD/PD) and number of regimens employed after disease progression following first-line chemotherapy were identified as prognostic factors for PPS. We suggest that the treatment course after disease progression following first-line chemotherapy significantly affects OS. We recommend that these results are validated to determine whether they can be generalized to larger populations.

\section{Acknowledgements}

We would like to thank Drs Tomohito Kuwako, Yosuke Miura, Yasuki Iwasaki, Shinichi Ishihara, Satoshi Tsuchiya and Satoru Watanabe for their assistance with this manuscript.

\section{References}

1. Siegel R, DeSantis C, Virgo K, et al: Cancer treatment and survivorship statistics, 2012. CA Cancer J Clin 62: 220-241, 2012.

2. Broglio KR and Berry DA: Detecting an overall survival benefit that is derived from progression-free survival. J Natl Cancer Inst 101: 1642-1649, 2009.

3. Soria JC, Massard C and Le Chevalier T: Should progression-free survival be the primary measure of efficacy for advanced NSCLC therapy? Ann Oncol 21: 2324-2332, 2010.

4. Reck M, von Pawel J, Zatloukal P, et al: Phase III trial of cisplatin plus gemcitabine with either placebo or bevacizumab as first-line therapy for nonsquamous non-small-cell lung cancer: AVAil. J Clin Oncol 27: 1227-1234, 2009.

5. Saad ED, Katz A and Buyse M: Overall survival and post-progression survival in advanced breast cancer: a review of recent randomized clinical trials. J Clin Oncol 28: 1958-1962, 2010.

6. Sundar S, Wu J, Hillaby K, Yap J and Lilford R: A systematic review evaluating the relationship between progression free survival and post progression survival in advanced ovarian cancer. Gynecol Oncol 125: 493-499, 2012.

7. Petrelli F and Barni S: Correlation of progression-free and post-progression survival with overall survival in advanced colorectal cancer. Ann Oncol 24: 186-192, 2013.

8. Hotta K, Kiura K, Fujiwara Y, et al: Role of survival post-progression in phase III trials of systemic chemotherapy in advanced non-small-cell lung cancer: a systematic review. PLoS One 6: e26646, 2011.

9. Hayashi H, Okamoto I, Morita S, Taguri M and Nakagawa K: Postprogression survival for first-line chemotherapy of patients with advanced non-small-cell lung cancer. Ann Oncol 23: $1537-1541,2012$
10. Imai H, Takahashi T, Mori K, et al: Individual-level data on the relationships of progression-free survival, post-progression survival, and tumor response with overall survival in patients with advanced non-squamous non-small cell lung cancer. Neoplasma 61: 233-240, 2014.

11. Mok TS, Wu YL, Thongprasert S, et al: Gefitinib or carboplatin-paclitaxel in pulmonary adenocarcinoma. N Engl J Med 361: 947-957, 2009.

12. Mitsudomi T, Morita S, Yatabe Y, et al; West Japan Oncology Group: Gefitinib versus cisplatin plus docetaxel in patients with non-small-cell lung cancer harbouring mutations of the epidermal growth factor receptor (WJTOG3405): an open label, randomised phase 3 trial. Lancet Oncol 11: 121-128, 2010.

13. Maemondo M, Inoue A, Kobayashi K, et al: Gefitinib or chemotherapy for non-small-cell lung cancer with mutated EGFR. N Engl J Med 362: 2380-2388, 2010.

14. Inoue A, Kobayashi K, Maemondo M, et al: Updated overall survival results from a randomized phase III trial comparing gefitinib with carboplatin-paclitaxel for chemo-naive non-small cell lung cancer with sensitive EGFR gene mutations (NEJ002). Ann Oncol 24: 54-59, 2013.

15. Nagai Y, Miyazawa H, Huqun, et al: Genetic heterogeneity of the epidermal growth factor receptor in non-small cell lung cancer cell lines revealed by a rapid and sensitive detection system, the peptide nucleic acid-locked nucleic acid PCR clamp. Cancer Res 65: 7276-7282, 2005.

16. Yatabe Y, Hida T, Horio Y, Kosaka T, Takahashi T and Mitsudomi T: A rapid, sensitive assay to detect EGFR mutation in small biopsy specimens from lung cancer. J Mol Diagn 8: 335-341, 2006.

17. Eisenhauer EA, Therasse P, Bogaerts J, et al: New response evaluation criteria in solid tumours: revised RECIST guideline (version 1.1). Eur J Cancer 45: 228-247, 2009.

18. Johnson KR, Ringland C, Stokes BJ, et al: Response rate or time to progression as predictors of survival in trials of metastatic colorectal cancer or non-small-cell lung cancer: a meta-analysis. Lancet Oncol 7: 741-746, 2006.

19. Hotta K, Fujiwara Y, Matsuo K, et al: Time to progression as a surrogate marker for overall survival in patients with advanced non-small cell lung cancer. J Thorac Oncol 4: 311-317, 2009.

20. Weir CJ and Walley RJ: Statistical evaluation of biomarkers as surrogate endpoints: a literature review. Stat Med 25: 183-203, 2006.

21. Fleischer F, Gaschler-Markefski B and Bluhmki E: A statistical model for the dependence between progression-free survival and overall survival. Stat Med 28: 2669-2686, 2009.

22. Foster NR, Qi Y, Shi Q, et al: Tumor response and progression-free survival as potential surrogate endpoints for overall survival in extensive stage small-cell lung cancer: findings on the basis of North Central Cancer Treatment Group trials. Cancer 117: 1262-1271, 2011

23. Berghmans T, Pasleau F, Paesmans M, et al; ELCWP: Surrogate markers predicting overall survival for lung cancer: ELCWP recommendations. Eur Respir J 39: 9-28, 2012.

24. Tsujino K, Kawaguchi T, Kubo A, et al: Response rate is associated with prolonged survival in patients with advanced non-small cell lung cancer treated with gefitinib or erlotinib. J Thorac Oncol 4: 994-1001, 2009.

25. Li X, Liu S, Gu H and Wang D: Surrogate end points for survival in the target treatment of advanced non-small-cell lung cancer with gefitinib or erlotinib. J Cancer Res Clin Oncol 138: 1963-1969, 2012.

26. Tomizawa Y, Fujita Y, Tamura A, et al: Effect of gefitinib re-challenge to initial gefitinib responder with non-small cell lung cancer followed by chemotherapy. Lung Cancer 68: 269-272, 2010. 